\title{
3D Interaction with the Desktop Bat
}

\author{
Anthony Steed and Mel Slater \\ Department of Computer Science, Queen Mary and Westfield College, Mile End Road, London, E1 4NS, UK
}

\begin{abstract}
Many applications now demand interaction with visualizations of $3 D$ scenes and data sets. Current flat $2 D$ displays are limited in their capacity to provide this not only by the display technology but the interaction metaphors and devices used. The Desktop Bat is a device that has 5 degrees of freedom whilst retaining the simplicity of use of a mouse. To use it for general $3 D$ interaction several metaphors were created for the tasks of navigation and cursor manipulation and a set of experiments were conducted to determine which metaphors were the most efficient in use. Of these metaphors, a velocity control metaphor was the best for navigation and a metaphor that applied rotations and translations relative to the eyepoint coordinate system was best for object control.
\end{abstract}

Keywords: 3D Interaction, Interaction Devices, Virtual Environments, Bat.

\section{Introduction}

There has been a great increase in the number of $3 \mathrm{D}$ computer aided design and data visualization systems in recent years. These typically rely on the use of $2 \mathrm{D}$ input devices to perform what are essentially six degrees of freedom tasks, with resulting complication in the user interface ${ }^{23,22}$. Immersive virtual reality techniques promise a more natural and intuitive interface to 3D environments, but the limitation of current technology makes them unsuitable for accurate or long term work. It is also not clear in which application contexts immersive virtual reality systems provide an advantage over flat $2 \mathrm{D}$ displays in terms of user efficiency. Therefore it is still important to consider 3D interaction within the desktop paradigm using multidimensional input devices.

The purpose of this paper is to introduce and evaluate metaphors for interaction with $3 \mathrm{D}$ environments using the Desktop Bat ${ }^{18}$. In the first section we will identify the main requirements for interaction within 3D environments and approaches previously used. Next we will describe metaphors that allow interaction with environments using the Desktop Bat. The fourth section section describes the experimental set-up used to evaluate each of the metaphors described and the results from these experiments are dis-

(C) The Eurographics Association 1995. Published by Blackwell Publishers, 108 Cowley Road, Oxford OX4 1JF, UK and 238 Main Street, Cambridge, MA 02142, USA. cussed in section five. Finally we consider these results and discuss ideas for future work.

\section{Requirements for Interaction}

The two basic tasks of 3D interaction are navigation and object placement. More complicated tasks such as deformation can be performed by the specification of one or more object positions ${ }^{18}$. For example a $3 \mathrm{D}$ input device can be used to specify a selection and then the device can be repositioned to specify the deformation.

\subsection{Object Positioning}

The task of positioning an object has six degrees of freedom with three for the location and three for the orientation.

With two degree of freedom devices being so widespread, many approaches have used mice and joysticks to control objects either directly using mode switches to enable translations and rotations in different directions, or through the use of virtual devices such as a virtual trackball 16, 7, 2, 3, 12 .

With the development of six degrees of freedom position sensors ${ }^{15}$, a more direct metaphor using hand 
location and orientation for object location and orientation becomes available. However it is not obvious that such a technique is ideal for long periods of interaction in virtual environments, with their accuracy limitations and tendency to be tiring to use ${ }^{1,25,24}$.

\subsection{Navigation}

Mackinlay et al. identify four main types of navigation 13 .

1. General movement,

2. Targeted movement,

3. Specified coordinate movement,

4. Specified trajectory movement.

We are concerned with the first type, unconstrained exploration of an environment, the other types of movement allow navigation to be determined by multiple object placements.

Once again, two degree of freedom input devices can be used for navigation, either directly or through virtual devices 17, 27. An example is using a mouse to point out on the display the required direction of movement and using a different mode to control rotations.

For movement over long distances velocity control can be used 10, 27, 6, 20. Velocity control allows rapid movement over large distances, but is inaccurate when approaching an object, though this problem can be overcome by use of a logarithmic approach technique 13 .

Virtual reality systems have made the idea of slaving the eyepoint to the position of the head popular $21,11,8,14$. However, because of the limited range of position trackers, the user is often constrained to stay within a small area and thus metaphors have to be used to navigate over longer distances 4, 5, 19 .

\section{Using the Desktop Bat for Interaction}

The Desktop Bat consists of a dome attached via three joints to a mouse base ${ }^{18}$. The dome rotates in three directions and combined with the planar location of the Desktop Bat this makes it a five degree of freedom device (see Figure 1). The Desktop Bat also has five buttons that are placed under the natural resting places of the fingers and thumb.

The conventions used in the following descriptions of the interaction metaphors are: the world coordinate system is defined by the axes $\mathrm{X}, \mathrm{Y}$ and $\mathrm{Z}$, where for exposition we are taking the vertical direction in the scene to be parallel to the $\mathrm{Z}$ axis; the eye coordinate system is defined by the axes $\mathrm{U}, \mathrm{V}$ and $\mathrm{N}$ where the

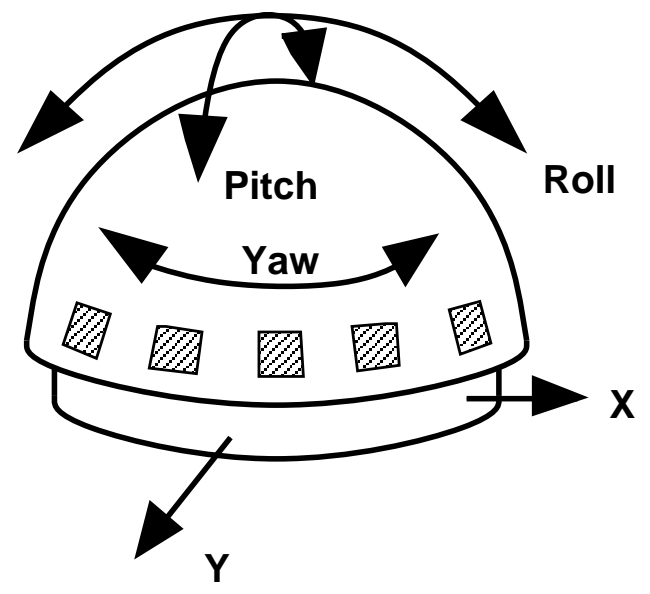

Figure 1: Degrees of freedom of the Desktop Bat

line of sight is along the $\mathrm{N}$ axis and up relative to the eye is along the $\mathrm{V}$ axis.

The Bat has five degrees of freedom, but the task of navigation requires 6 degrees of freedom, with 3 required to specify the location and 3 for the orientation of the eyepoint. Three metaphors that allow general navigation are:

1. Hand on Eye. In this metaphor rotations of the Bat cause rotations of $\mathrm{U}, \mathrm{V}$ and $\mathrm{N}$ axes. For example roll of the Bat corresponds to rotation of the $\mathrm{U}$ and $\mathrm{V}$ axes around the $\mathrm{N}$ axis. Planar movement of the Bat moves the eyepoint in the plane UN, see Figure 2 .

2. Simple Camera. In this case the eyepoint is regarded as a video camera. Yaw of the Bat corresponds to rotation of the camera about the $\mathrm{Z}$ axis. Pitch of the Bat corresponds to the angle between the $\mathrm{N}$ axis and the XY plane. As before planar movement of the Bat moves the eyepoint in the UN plane, see Figure 3. Roll has no effect with this metaphor.

3. Hand on Eye with Velocity. This is similar to the hand on eye metaphor, except the planar displacement of the Bat is taken as velocity in the UN plane.

These metaphors are similar to Ware and Osborne's eyeball in hand and flying vehicle metaphors for navigation 26 in that they directly control the eyepoint with the input device. However because of the different designs of the input devices used, the manner in which orientation is controlled is different.

Picking and moving objects is also a 6 degrees of freedom task, though here the situation is more complicated because the two tasks, picking and placement 


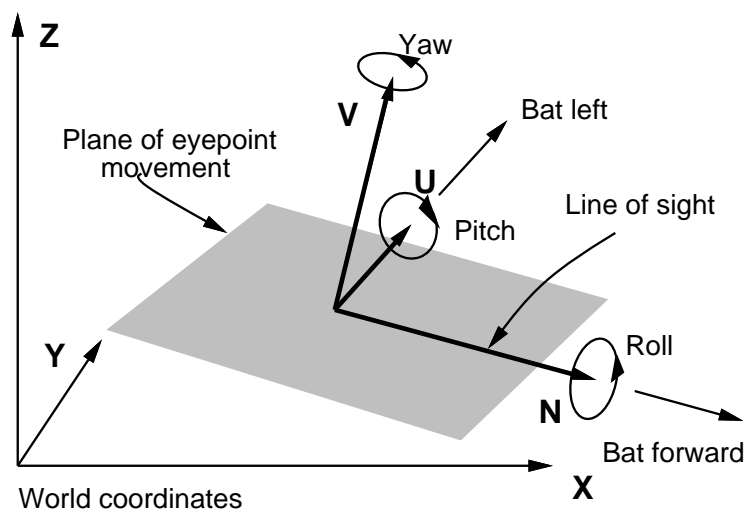

Figure 2: Hand on Eye Metaphor

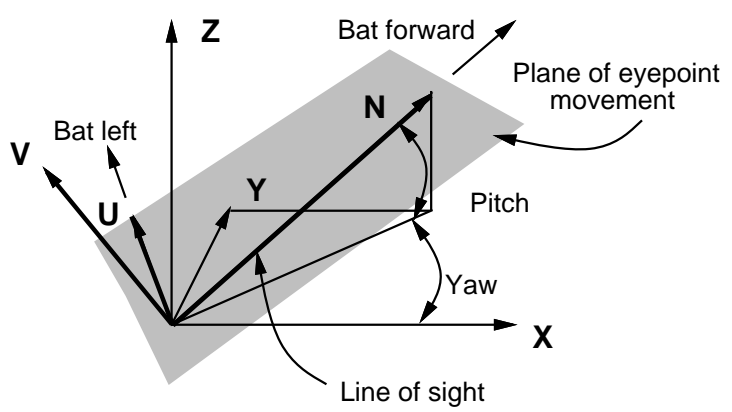

Figure 3: Simple Camera Metaphor

of objects, result in different demands on the metaphor used.

The Bat controls a 3D cursor and when an object is picked it becomes fixed relative to the cursor. Hence rotation of the bat causes the object to rotate about the cursor's center. The cursor's coordinate system is defined by the axes $\mathrm{P}, \mathrm{Q}$ and $\mathrm{R}$ where $\mathrm{P}$ is the direction in which the cursor is pointing and $\mathrm{R}$ is the cursor's up vector. The metaphors for moving the cursor are:

1. Relative to Cursor. Rotations of the Bat cause rotation of the PQR system. For example roll of the Bat causes roll of the $\mathrm{Q}$ and $\mathrm{R}$ axes about the $\mathrm{P}$ axis. Planar movement of the Bat causes movement of the object in the plane PQ, see Figure 4 .

2. Relative to world. Rotations of the Bat cause rotation of the PQR system. However when the Bat is moved forward the PQR system moves along the vector defined by the projection of the axis $\mathrm{P}$ into the plane XY, see Figure 5.

3. Relative to Eye. In this metaphor rotating the Bat causes rotation of the PQR system in the UVN system. For example yaw of the Bat corresponds to rotation of the PQR system around the $\mathrm{V}$ axis.

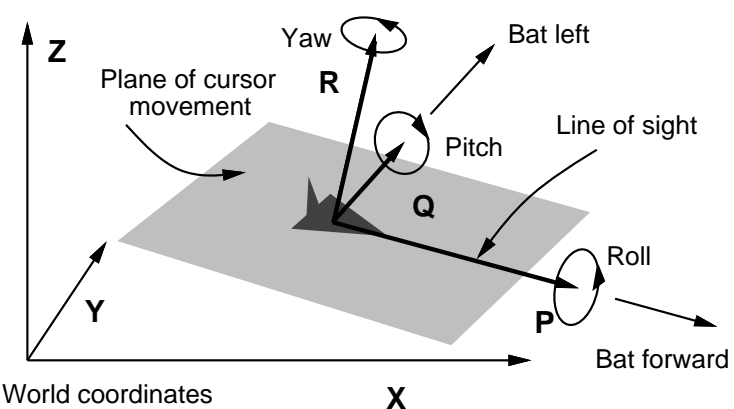

Figure 4: Relative to Cursor Metaphor

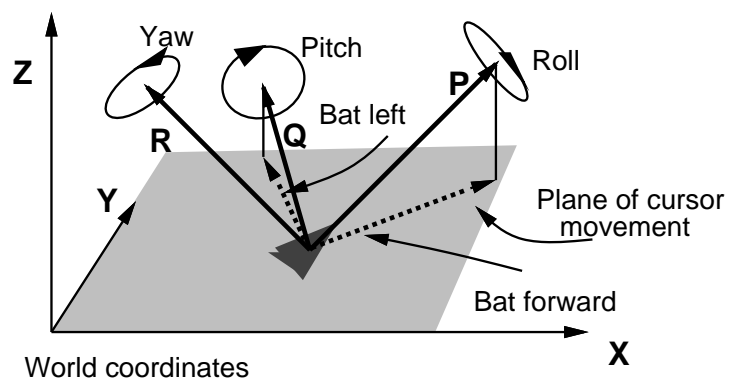

Figure 5: Relative to World Metaphor

Planar movement of the Bat then moves the object in the plane defined by $\mathrm{U}$ and $\mathrm{N}$, see Figure 6 .

The Desktop Bat's buttons allow the definition of simple gestures to effect changes in the interaction mode. The most important gesture uses a single button that acts as a clutch. The clutch disables the Bat so that it can be used in a relative mode, or so that the hand can be reoriented to a flat, comfortable position. The other required gestures, each of which uses two buttons, are: to swap between eyepoint and cursor control; to enable and disable object picking; to recall the cursor to a position in front of the eye; and finally to enable translations of the eye or cursor in a direction orthogonal to that usually allowed by the metaphor. For example, to move vertically instead of horizontally. The gestures were each designed with a simple metaphor in mind. For example the gesture to pick objects uses the buttons under the thumb and first finger, which corresponds to a pinching action.

There are nine possible combinations of these navigation and manipulation metaphors. Their relative utility for different applications is an empirical question and the next section describes an experiment designed to answer this. 


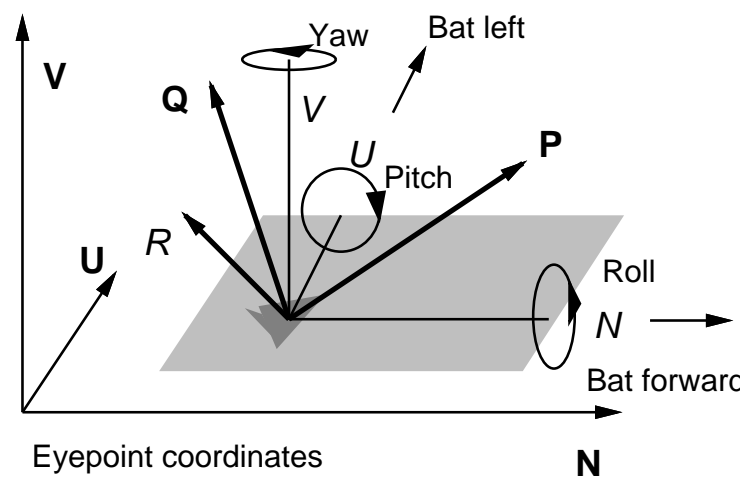

Figure 6: Relative to Eye Metaphor

\section{Experimental Design}

Different applications place different emphasis on the use of the input device for navigation, picking and placement of objects. Accordingly three scenarios were designed each with different primary tasks for the user to perform(see Plates 1-3):

1. Find. Involved the user searching for three objects on a plane and then placing them on a table accurately enough to lock them into position. This places emphasis on the ability to place objects with the Desktop Bat.

2. Data. This scenario consisted of a wireframe cube inside of which there were several variously shaped and coloured polyhedra. The user had to remove each of the eight yellow cubes from the wireframe cube, testing the ability to pick objects.

3. Maze. This scenario consisted of three tunnels leading away from a central room. The task was to navigate along each tunnel to a room at the other end, and in that room to pick up the object resting on the table and to bring it back to the central room. This scenario was primarily to judge navigation ability.

To evaluate the metaphors several measurements were taken automatically during the experiments. The efficiency of the navigation metaphor is indicated by the Eye Time, the time spent manipulating the eye; Eye Changes, the number of times the clutch was pressed when in eye mode; and Eye Adjust Time, the time spent using the clutch whilst in navigation mode. The overall efficiency of the cursor metaphor is shown by Cursor Time, the time spent manipulating the cursor; Cursor Changes, the number of times the clutch was pressed when in cursor mode; and Cursor Adjust Time, the time spent with the clutch depressed whilst in cursor mode. The times spent picking and placing objects were also of interest, and these are recorded as Hold time and Pick time. The number of times each gesture was used and the length of time spent using the mode which that gesture enabled were also recorded to see if the metaphors had a effect on them. These are: Number of Recalls, the number of times the cursor was recalled in front of the eye; Cursor-Eye Mode Swaps, the number of mode changes between navigation and cursor control; Cursor Vertical Move Time, the time spent moving the cursor vertically; and finally Eye Vertical Move Time, the time spent moving the eye vertically. Finally Total time taken to complete the task was recorded.

The standard analysis of variance model for each of these variables was assumed:

$$
y_{i j k l}=\mu+\alpha_{i}+\beta_{j}+\gamma_{k}+\delta_{i j}+\epsilon_{i j k l}
$$

Where $y_{i j k l}$ is an instance of one of the dependent variables, $\alpha_{i}$ the the effect of being in the $i^{t h}$ eye metaphor, $\beta_{j}$ is the effect of being in the $j^{\text {th }}$ cursor metaphor, $\gamma_{k}$ is the effect of being in the $k^{t h}$ scenario, $\delta_{i j}$ is an interaction effect between the eye and cursor metaphors, and $\epsilon_{i j k l}=\operatorname{Normal}\left(0, \sigma^{2}\right)$. The index $l$ refers to the repetition number for each combination of scenarios and metaphors and $\mu$ is the general mean.

A Latin Square distribution of metaphors and scenes was devised. Forty-five people took part in the experiment, allowing five repetitions of each scenario/metaphor combination. The subjects were recruited from amongst the students and staff of Queen Mary and Westfield College's Computer Science Department and Computer Services Centre. All had experience using a mouse-based computer interface. The system was implemented on a Silicon Graphics IRIS Indigo Elan using the GL graphics library.

The hypotheses of the experiment were as follows:

1. That the velocity control metaphor for the eye would be best in terms of time taken to complete navigation tasks.

2. The relative to cursor metaphor would be best for picking up objects.

3. The relative to eye and relative to world metaphors would be superior to the relative to cursor metaphor for placing objects.

4. Overall the relative to eye metaphor would be best for the general task of object manipulation.

\section{Results}

In the following analysis of the results in the first instance an analysis of variance (ANOVA) was performed on the data. From the results of this analysis the significant factors were determined and these factors were analysed further. The scenario will be a significant factor for each dependent variable because of 
the different tasks performed in each case and as this is not relevant in the comparison of the metaphors it will be ignored.

The results show that the velocity control metaphor is best for navigation and the relative to eye is best for object manipulation.

In the following description, dependent variables for which there were no significant factors, except those directly relevant to the hypothesis, are not presented.

\subsection{Eye Time}

No factor was significant in the ANOVA. In particular the eye metaphor was not significant contrary to what was expected, as shown in Table 1.

\subsection{Cursor time}

The mean times by cursor metaphor shows what was expected, but the difference in the means was not statistically significant, see Table 2 .

\subsection{Eye adjust time}

In this case both independent factors and the interaction effect were significant at the $1 \%$ level in the ANOVA. The mean times for each eyepoint metaphor, shown in Table 1, suggest that the subjects spent less time adjusting the eye when using the velocity metaphor. The mean times for each cursor metaphor, shown in Table 2, are interesting as they indicate that the subjects spent significantly less time adjusting the eye if they had the relative to eye cursor metaphor. The reason for this may be that with the relative to eye metaphor the subjects did not need to move the eyepoint to a specific position. For picking objects it was advantageous to be level with the object in the relative to world metaphor. For placing objects it was advantageous to be above the placement area and looking almost directly down at it. With the relative to eye metaphor there is little advantage in such accurate placing of the eye.

The significance of the interaction effect is harder to understand. To solve the linear model most components in the interaction effect were aliased to zero, but the resulting model tells us that when using the simple camera metaphor for the eye, the best accompanying cursor metaphor is the relative to cursor with the other two cursor metaphors having little to distinguish between them.

\subsection{Cursor adjust time}

Cursor metaphor was significant in the ANOVA at the $10 \%$ level. The mean times for each object metaphor, shown in Table 2, suggest that the relative to world metaphor requires most use of the clutch and the relative to eye requires least.

\subsection{Eye changes}

Both independent factors and the interaction effect were significant in the ANOVA. The analysis of times provides the same relationships between the metaphors as those for eye adjust time as would be expected, the number of times the clutch is used and the actual amount of time spent using it are directly related.

\subsection{Pick time}

No factor was significant in the ANOVA. However looking at the means for each cursor metaphor, shown in Table 2, indicates what was expected.

\subsection{Hold time}

Both independent factors and the interaction effect were significant in the ANOVA. Cursor metaphor was significant at the $1 \%$ level, eye metaphor at $5 \%$ level and interaction effect at the $10 \%$. The results show what was expected for the cursor metaphor, as shown in Table 2. The mean times for each eye metaphor, (Table 1), indicate that the hand on eye and velocity metaphors give an advantage over the simple camera metaphor.

The model also suggests that if using the simple camera metaphor for the eye, the best accompanying cursor metaphor is the relative to world, followed by relative to eye, then relative to cursor.

\subsection{Cursor vertical move time}

Interaction effect and cursor metaphor were both significant at the $1 \%$ level in the ANOVA. The mean times for each cursor metaphor, see Table 2, show that the relative to world metaphor required the vertical mode to be used frequently because it is necessary in order to change the horizontal plane in which the cursor moves. The relative to eye metaphor also requires the vertical mode to pick or place an object not in the plane of the eye.

The model also suggests that if using the simple camera metaphor for the eye, the best accompanying cursor metaphor is the relative to world, followed by relative to cursor, then relative to eye. 


\begin{tabular}{lccc} 
& \multicolumn{3}{c}{ Dependent Variables } \\
\cline { 2 - 4 } Eye Metaphor & Eyepoint Time & Eye Adjust Time & Hold Time \\
\hline Hand On Eye & $361.5 \pm 196.4$ & $74.6 \pm 67.8$ & $122.0 \pm 62.2$ \\
\hline Simple Camera & $360.3 \pm 196.3$ & $73.5 \pm 53.7$ & $184.9 \pm 148.8$ \\
\hline Hand on Eye with Velocity & $344.6 \pm 218.9$ & $36.0 \pm 35.3$ & $147.0 \pm 127.9$ \\
\hline
\end{tabular}

Table 1: Mean and Standard deviations in seconds of dependent variables for each eyepoint metaphor

\begin{tabular}{|c|c|c|c|c|c|c|}
\hline \multirow[b]{2}{*}{ Cursor Metaphor } & \multicolumn{6}{|c|}{ Dependent Variables } \\
\hline & Cursor Time & $\begin{array}{c}\text { Eye Adjust } \\
\text { Time }\end{array}$ & $\begin{array}{c}\text { Cursor Adjust } \\
\text { Time }\end{array}$ & Pick Time & Hold Time & $\begin{array}{c}\text { Cursor Vert. } \\
\text { Time }\end{array}$ \\
\hline Relative to Cursor & $229 \pm 208$ & $78.2 \pm 65.0$ & $8.8 \pm 9.9$ & $49.0 \pm 77.6$ & $180 \pm 154$ & $10.1 \pm 18.4$ \\
\hline Relative to World & $238 \pm 136$ & $70.2 \pm 58.6$ & $13.2 \pm 12.6$ & $70.2 \pm 73.6$ & $168 \pm 109$ & $56.0 \pm 61.1$ \\
\hline Relative to Eye & $163 \pm 120$ & $58.6 \pm 31.4$ & $6.0 \pm 7.4$ & $57.8 \pm 90.6$ & $105 \pm 62$ & $24.5 \pm 20.4$ \\
\hline
\end{tabular}

Table 2: Mean and Standard deviations in seconds of dependent variables for each cursor metaphor

\subsection{Eye vertical move time}

Scenario and interaction effect were significant in ANOVA, interaction effect at the $1 \%$ level. The results for the intercation effect show that when using the simple camera metaphor for the eye then the best cursor metaphor to combine it with is the relative to world, then the relative to cursor, and then the relative to eye.

\section{Conclusions}

The results are consistent with the hypotheses, but for reasons not always anticipated.

The velocity control metaphor is best for navigation, though this is not due to the actual time spent moving, but the lesser amount of time spent using the clutch with this metaphor. This is understandable, to navigate long distances with the simple camera or hand on eye metaphor requires the clutch to be used whenever the bat has been pushed as far as the arm will reach. When using the velocity control metaphor the clutch was only used when turning a corner around which the subjects could not see, in which case they would stop, turn and then speed up again. The velocity control metaphor also has an advantageous effect on the time spent holding an object, though the reason for this has not been determined.

For the object manipulation metaphor the results have confirmed that different metaphors are best for the two tasks of picking and placing. The relative to cursor metaphor was marginally better for picking objects when looking at the mean times for object placement, and the relative to eye was best for placing objects. The relative to world metaphor did not give as much of an advantage for placing objects as originally thought. Overall the relative to eye metaphor would be the best choice due to the greater difficulty of placing objects compared to picking them. This is confirmed by the analysis of the cursor adjust times and cursor changes that would seem to suggest that orientating the cursor is easier with the relative to eye metaphor. The cursor vertical move time indicates that the relative to eye metaphor requires a lot of vertical movement, but this time is a part of the total time spent using the cursor and so does not suggest a poorer overall performance for this metaphor.

More succinctly, the velocity control metaphor is best for navigation and the relative to eye is best for object manipulation.

\section{Discussion and Further Work}

The Desktop Bat has advantages over some other devices in that it rests on the desktop and does not lead to fatigue from having to hold out a $3 \mathrm{D}$ position sensor. It also can be used as a normal mouse for applications that require switching between $3 \mathrm{D}$ and $2 \mathrm{D}$ environments. The lack of a sixth degree of freedom is not a problem since the metaphors discussed earlier allow general movement in $3 \mathrm{D}$ environments. In fact object placement may be easier if not all the degrees of freedom of a 6 degrees of freedom device are en- 
abled simultaneously ${ }^{24}$. For this reason a comparison between the Desktop Bat and a device such as the SpaceBall would be interesting.

There are many more aspects of the use of the Desktop Bat to be investigated. Improved velocity control metaphors that use a non-linear scaling between device movement and velocity is one area ${ }^{27,20}$. Another that was highlighted as a result of the experiments is the use of the clutch in the opposite manner, that is to enable rotations and translations rather than disable them. Several subjects expected this to happen and when they used the clutch in the same manner as a ratchet is used, they started moving in the opposite direction to that which they expected.

Several applications are being developed that will use the Desktop Bat for 3D tasks. It has been suggested that some applications might benefit from the use of two Desktop Bats with one for navigation and one for object selection.

A more advanced version of the Desktop Bat than reported in ${ }^{18}$ has been developed ${ }^{9}$, unfortunately this was not available in time to be used during the experiments.

\section{Acknowledgements}

Thanks go to Tim Barnes for the construction of the prototype Desktop Bat used in the experiments and to Derek Coppen for valuable assistance in interfacing and maintaining the Desktop Bat. Anthony Steed is supported by a UK EPSRC studentship.

\section{References}

1. N. I. Badler, K. H. Manoochechri, and D. Baraff. Multi-dimensional input techniques and articulated figure positioning by multiple contraints. In F. Crone and S. M. Pizer, editors, Proc. 1986 ACM Workshop on Interactive 3D Graphics, Chapel Hill, NC, October 22-24, pages 151169. ACM Press, 1986.

2. E. A. Bier. Skitters and jacks: Interactive 3D positioning tools. In F. Crone and S. M. Pizer, editors, Proc. 1986 ACM Workshop on Interactive 3D Graphics, Chapel Hill, NC, October 2224, pages 183-196. ACM Press, 1986.

3. E. A. Bier. Snap-dragging in three dimensions. Computer Graphics, 24(2):193-203, 1990. Special Issue on Symposium on Interactive 3D Graphics.

4. C. Blanchard, S. Burgess, Y. Harvill, J. Lanier, A. Lasko, M. Oberman, and M. Teitel. Reality built for two: A virtual reality tool. Computer Graphics, 24(2):35-36, March 1990.
5. K. Böhm, W. Hübner, and K. Väänänen. Given: gesture driven interactions in virtual environments. A toolkit approach to 3D interactions. In Informatique 92: Interface to Real and Virtual Worlds, Montpellier France, 23-27 March, pages 243-254, 1992.

6. D. Chapman and C. Ware. Manipulating the future: Predictor based feedback for velocity control invirtual environment navigation. Computer Graphics. Special Issue 1992 Symposium on Interactive 3D Graphics, pages 63-66, 1992.

7. M. Chen, S. J. Mountford, and A. Sellen. A study in interactive $3-\mathrm{D}$ rotation using $2-\mathrm{D}$ devices. Computer Graphics, 22(4):121-129, August 1988.

8. J. C. Chung, M. R. Harris, F. P. Brooks, H. Fuchs, M. T. Kelley, J. Hughes, M. Ouh-Young, C. Cheung, R. L. Holloway, and M. Pique. Exploring virtual worlds with head-mounted displays. SPIE Vol. 1083 Three-Dimensional Visualization and Display Technologies, pages 42-52, 1989.

9. D. Coppen. The optical desktop bat. Internal memo: Department of Computer Science, Queen Mary and Westfield College, University of London, November 1993.

10. S. S. Fisher. Virtual interface environments. In Brenda Laurel, editor, The Art of HumanComputer Interface Design. Addison Wesley, 1990.

11. S. S. Fisher, M. McGreevy, J. Humphries, and W. Robinett. Virtual environment display system. Proc. 1986 ACM Workshop on Interactive $3 D$ Graphics, Chapel Hill, NC, October 22-24, pages 77-87, 1986.

12. J. Hultquist. A virtual trackball. In A. S. Glassner, editor, Graphics Gems, pages 462-463. Academic Press, 1990.

13. J. D. Mackinlay, S. K. Card, and G. G. Robertson. Rapid controlled movement through a virtual 3D workspace. Computer Graphics, 24(4):171-176, August 1990.

14. M. McKenna. Interactive viewpoint control and three-dimensional operations. Computer Graph$i c s$, pages 53-56, 1992. Special Issue on Symposium on Interactive 3D Graphics.

15. K. Meyer, H. L. Applewhite, and F. A. Biocca. A survey of position trackers. Presence: Teleoperators and Virtual Environments, 1(2):173-200, 1992.

16. G. M. Nielson and D. R. Olsen Jr. Direct manipulation techniques for $3 \mathrm{D}$ objects using $2 \mathrm{D}$ 
locator devices. In F. Crone and S. M. Pizer, editors, Proc. 1986 ACM Workshop on Interactive 3D Graphics, Chapel Hill, NC, October 2224, pages 175-182. ACM Press, 1986.

17. G. G. Robertson, S. K. Card, and J. D. Mackinlay. The cognitive coprocessor architecture for interactive user interfaces. In Proceedings UIST'89, pages 10-18, 1989.

18. M. Slater and A. Davidson. Liberation from flatland: 3D interaction based on the desktop bat. In EUROGRAPHICS '91, pages 209-221. Elsevier Science Publishers B.V. (North-Holland), 1991.

19. M. Slater, A. Steed, and M. Usoh. The virtual treadmill: A naturalistic metaphor for navigation in immersive virtual environments. In M. Göbel, editor, First Eurographics Workshop on Virtual Environments, Polytechnical University of Catalonia, September 7, pages 71-83, 1993.

20. D. Song and M. Norman. Nonlinear interactive motion control techniques for virtual space navigation. In Proceedings of IEEE 1993 Virtual Reality Annual International Symposium, VRAIS '93, Piscataway, NJ., pages 111-117, 1993.

21. I. E. Sutherland. A head-mounted three dimensional display. In Proc. AFIPS Fall Joint Computer Conference, 33, pages 757-764, 1968.

22. Virtus Corporation. Virtus walkthrough demo $1.3,1990$.

23. VPL Research. Swivel 3D Professional, 1991.

24. C. Ware. Using hand position for virtual object placement. Visual Computer, 6(5):245-253, 1990.

25. C. Ware and D. R. Jessome. Using the bat: A sixdimensional mouse for object placement. IEEE Computer Graphics and Applications, pages 6570, November 1988.

26. C. Ware and S. Osborne. Exploration and virtual camera control in virtual three dimensional environments. Computer Graphics, 24(2):175-183, March 1990.

27. C. Ware and L. Slipp. Using velocity control to navigate 3D graphical environments: A comparison of three interfaces. In Proceedings of the $\mathrm{Hu}$ man Factors Society 35th Annual Meeting, 1991. 